\title{
Low-mass X-ray binaries in the bulge of the Milky Way
}

\author{
M. Revnivtsev ${ }^{1,2,3}$, A. Lutovinov ${ }^{2}$, E. Churazov ${ }^{1,2}$, S. Sazonov ${ }^{1,2}$, M. Gilfanov ${ }^{1,2}$, S. Grebenev ${ }^{2}$, and R. Sunyaev ${ }^{1,2}$ \\ 1 Max-Planck-Institute für Astrophysik, Karl-Schwarzschild-Str. 1, 85740 Garching bei München, Germany \\ e-mail: mikej@mpa-garching.mpg.de \\ 2 Space Research Institute, Russian Academy of Sciences, Profsoyuznaya 84/32, 117997 Moscow, Russia \\ 3 Excellence Cluster Universe, Technische Universität München, Garching, Germany
}

Received 2 May 2008 / Accepted 21 July 2008

\begin{abstract}
We study the population of low-mass X-ray binaries (LMXBs) in the Galactic bulge using the deep survey of this region by the IBIS telescope aboard the INTEGRAL observatory. Thanks to the increased sensitivity with respect to previous surveys of this field, we succeeded in probing the luminosity function (LF) of LMXBs down to $\sim 7 \times 10^{34} \mathrm{erg} / \mathrm{s}$ in the 17-60 keV energy band. The slope of the $17-60 \mathrm{keV}$ LF in the $10^{35}-10^{37} \mathrm{erg} / \mathrm{s}$ range is $\mathrm{d} \log N / \mathrm{d} \log L=-0.96 \pm 0.20$. At these low luminosities, the spectra of LMXBs between 2 and $60 \mathrm{keV}$ are similar to that of the $\mathrm{Crab}$, and therefore nearly equal amounts of energy are released in the standard $(2-10 \mathrm{keV})$ and hard $(17-60 \mathrm{keV}) \mathrm{X}$-ray bands. This implies that the LMXB LF is flat both in the $17-60 \mathrm{keV}$ and $2-10 \mathrm{keV}$ energy bands over the $10^{35}-10^{37} \mathrm{erg} / \mathrm{s}$ luminosity range. This further suggests that the faint end of the $2-10 \mathrm{keV} \mathrm{LF}$ is significantly flatter than its bright end (above $10^{37} \mathrm{erg} / \mathrm{s}$ ), where a slope of $\mathrm{d} \log N / \mathrm{d} \log L \sim-1.8$ was previously measured. We discuss the origin of this flattening. We also demonstrate that the spatial distribution of persistent LMXBs in the Galactic center/Galactic bulge region is consistent with a model of stellar mass distribution that includes the nuclear stellar disk component in the innermost degree of the Galaxy. The spatial distribution of transient LMXBs detected in the Galactic center region indicates an increased fraction of transient sources in the innermost degree of the Galaxy with respect to outer regions.
\end{abstract}

Key words. binaries: general - stars: luminosity function, mass function - Galaxy: bulge - X-rays: binaries - X-rays: general

\section{Introduction}

Low-mass X-ray binaries (LMXBs) are binary systems with a compact object that accretes matter from a low-mass optical companion star filling its Roche lobe (see e.g. van den Heuvel 1975). Ways of forming and the evolution of LMXBs are important questions of X-ray astronomy (see discussions of these issues in e.g., Webbink et al. 1983; Lipunov et al. 2007; Belczynski et al. 2008).

The shape of the luminosity function (LF) of LMXBs may provide important information about their long-term evolution. In particular, Postnov \& Kuranov (2005) note that the observed shape of the LMXB LF (assuming that the X-ray luminosity of the compact object is directly proportional to the mass transfer rate in the binary system) depends on the distribution of the masses of optical donor stars in LMXBs, which is in turn intimately related to the mechanism of removal of the angular momentum of the binary system. They suggested that the flattening of the LMXB LF at luminosities $L_{\mathrm{x}}<2 \times 10^{37} \mathrm{erg} / \mathrm{s}$ found in a number of studies (Primini et al. 1993; Gilfanov 2004; Voss \& Gilfanov 2006, 2007) might correspond to the transition from the magnetic stellar wind braking to the gravitational wave braking mechanism for removing the binary system angular momentum (Postnov \& Kuranov 2005). Confirmation of this idea would provide additional evidence for the action of gravitational braking mechanisms in LMXBs and enable one to select those binary systems effectively emitting gravitational waves for the next generation of gravitational wave detectors. It is therefore interesting and important to check whether the break in the LMXB LF is present and to continue the LF to lower luminosities.
Using LF as a tool for studying populations of X-ray sources in nearby galaxies is only possible with instruments that have high sensitivity and angular resolution. First attempts to estimate the LF of bright X-ray binaries were made with the Einstein/HEAO2 observatory (see e.g. van Speybroeck et al. 1979; Trinchieri \& Fabbiano 1991) more than twenty years ago. Studying LMXB LF in galaxies has now become quite common due to the unprecedented capabilities of the Chandra X-ray Observatory. However, even this powerful observatory does not allow one to probe the faint end $\left(L_{\mathrm{x}}<10^{36-37} \mathrm{erg} / \mathrm{s}\right)$ of the LMXB LF in the majority of nearby galaxies. This is only feasible for the nearest galaxies, like M31 (Trinchieri \& Fabbiano 1991; Primini et al. 1993; Voss \& Gilfanov 2007).

The Milky Way is a unique galaxy where we can detect X-ray objects with the lowest possible luminosities. However, because of the large angular size of the Galaxy and the different and often poorly known distances to Galactic X-ray sources, it is not easy to construct the LF of LMXBs in the Milky Way. First of all, one needs to cover a large fraction of the sky. However, focusing X-ray telescopes usually have small fields of view ( 10 arcmin) and thus cannot cover a considerable part of the Galaxy within a reasonable time. Even the region of maximal concentration of Galactic LMXBs - the Galactic bulge - is too large for focusing X-ray telescopes.

The Galactic center/Galactic bulge region is most suitable for studying Galactic LMXBs because the majority of sources are spatially concentrated and are located at nearly the same distance from the Sun. Skinner (1993) reconstructed the surface density map of X-ray sources in the Galactic bulge region using a compilation of observational campaigns by different 
observatories. The population of Galactic bulge X-ray sources was also studied with the exceptionally wide field-of-view ART-P coded-mask telescope aboard the GRANAT observatory (Grebenev et al. 1996).

Grimm et al. (2002) used data of the All-Sky Monitor (ASM) of the RXTE observatory in the $2-10 \mathrm{keV}$ energy band to construct the luminosity function of Galactic LMXBs with luminosities $L_{\mathrm{x}} \gtrsim 10^{36} \mathrm{erg} / \mathrm{s}$. Later, Gilfanov (2004) studied the populations of LMXBs in the Milky Way and nearby galaxies and showed that the LMXB LF has a break at a luminosity $L_{\mathrm{x}} \sim 2 \times 10^{37} \mathrm{erg} / \mathrm{s}$, below which it significantly flattens. Similar flattening of the LMXB LF was found by Primini et al. (1993) for the population of sources in the M31 galaxy. Recent studies of a number of galaxies performed with the Chandra observatory confirmed such a break in the LMXB LF in the M 31 galaxy (Kong et al. 2003; Voss \& Gilfanov 2007) and indicated the presence of a break for the Cen A galaxy (Voss \& Gilfanov 2006). No breaks, however, were detected by Kim et al. (2006) in the LF of sources in NGC 3379 and NGC 4278.

To make a significant improvement in the statistical study of LMXBs in our Galaxy and independently measure the faint end of the LMXB LF, one needs a large field-of-view instrument with increased (in comparison with previous ones) sensitivity. It is also necessary to use a hard X-ray energy band to avoid contamination by large numbers of nearby, soft X-ray emitting stars, as was the case with the ROSAT all-sky survey (e.g. Voges et al. 1999). This is exactly what is provided by the coded-mask telescopes of the INTEGRAL observatory (Winkler et al. 2003). Deep observations of the Galaxy performed with INTEGRAL/IBIS over five years of its operation have allowed us to increase the sensitivity to point sources by an order of magnitude in comparison with the RXTE/ASM survey. This allows us to probe the faint end of the LF of Galactic LMXBs for the first time.

\section{Sample of sources and data analysis}

To avoid problems with unknown source distances and consequently unknown source luminosities, we do not use the whole INTEGRAL survey of the Milky Way here and restrict ourselves to the Galactic center/Galactic bulge region where LMXBs are strongly concentrated (see e.g., Skinner 1993; Grebenev et al. 1996; Grimm et al. 2002; Lutovinov et al. 2005).

The INTEGRAL observatory has spent a lot of time observing the Galactic center (Galactic bulge) region over the first five years of its operations (see e.g., Revnivtsev et al. 2004; Kuulkers et al. 2007). Among the INTEGRAL instruments, the IBIS telescope best meets the requirements of the present study: it has high sensitivity for detecting point sources and a wide field of view $\left(\sim 28^{\circ} \times 28^{\circ}\right)$. Due to the hard energy band of IBIS $(>17 \mathrm{keV})$, it is less sensitive to sources that have high luminosities in the standard 2-10 keV energy band (since such sources have very soft spectra that do not continue to hard X-rays), but it is very effective in detecting low-luminosity objects (which typically have hard spectra). The sensitivity achieved by now with respect to point sources in the Galactic center region is typically $0.5 \mathrm{mCrab}$ in the $17-60 \mathrm{keV}$ energy band (see e.g., Krivonos et al. 2007b)

For the present study we selected all sources detected by INTEGRAL in the Galactic center (Galactic bulge) region listed in the catalog of Krivonos et al. (2007b) and added INTEGRAL/IBIS data that became available since the publication of this catalog. These additional data allowed us to increase the accuracy of source flux measurements and to add one newly detected source, IGR J17586-2129, to the sample.

We only considered those sources located within the elliptical region around the Galactic center with axes $|l|<10.7^{\circ},|b|<$ $5.1^{\circ}$ and having fluxes higher than $0.64 \mathrm{mCrab}$ in the $17-60 \mathrm{keV}$ energy band, which typically ensures the source detection with statistical significance more than $8 \sigma$. This conservative detection threshold prevents any problems with possible systematical uncertainties in the image reconstruction and with variations in the sensitivity over the region of our interest.

Due to the anticipated concentration of LMXBs in the Galactic bulge, we assume that all sources that are not known to be high-mass X-ray binaries, cataclysmic variables, or extragalactic sources are LMXBs. It is possible that a detailed study of some faint sources (typically with luminosities $L<10^{35} \mathrm{erg} / \mathrm{s}$ ) from our sample will reveal their non-LMXB nature. Therefore, we are probably somewhat overestimating the faint end of the LMXB LF.

From the preliminary source list we have filtered out:

1. HMXBs. High-mass X-ray binaries (HMXBs) are young objects, which in our Galaxy are concentrated in the regions of recent star formation - the spiral arms. The spatial distribution of Galactic HMXBs has been studied by Grimm et al. (2002) in soft X-rays and by Lutovinov et al. (2005, 2007) in hard X-rays ( $>20 \mathrm{keV})$ with the INTEGRAL observatory. Due to the low star-formation rate in the Galactic bulge we can anticipate that HMXBs should not be a dominant population of bright X-ray sources there. However, we might detect some HMXBs located in the Galactic disk, projected on the bulge in the sky plane. We excluded from our analysis all sources that are known or supposed to be HMXBs, for example so-called supergiant fast X-ray transients (SFXTs) and X-ray pulsars. These sources include EXO 1722-363, IGR J17407-2808, XTE J1743-363, IGR J17544-2619, IGR J17391-3021 (XTE J1739-302), AX J1749.1-2733, AX J1749.2-2725, and IGR J18027-2016. Note that most of them have a transient nature.

2. Extragalactic. We also excluded extragalactic sources. Within the region of our study there are only two known extragalactic sources - active galactic nuclei GRS 1734-292 and IGR J17488-3253. According to the all-sky averaged AGN number-flux function in hard X-rays (Krivonos et al. 2007b), we should detect $\sim 2$ AGNs above the adopted flux limit of $0.64 \mathrm{mCrab}$ within the area of our study ( $\sim 171$ sq. deg). This indicates that most likely we already know of all the extragalactic sources with fluxes of $>0.64 \mathrm{mCrab}$ within the considered field. The probability that there are 2 or more unidentified extragalactic sources in this region is $\sim 0.14$, and we do not expect unknown extragalactic sources to considerably contribute to the measured number-flux function of sources within the bulge region.

3. Other sources. We removed cataclysmic variables, detected in the considered region. We removed from our sample the source IGR J17402-3656, which is associated with the nearby open star cluster NGC 6400 and may be composed of multiple point sources; the source IGR J17456-2901, which is similarly associated with the collective emission of the nuclear stellar cluster (Bélanger et al. 2006; Krivonos et al. 2007a); and the source IGR J17475-2822 representing the emission of the molecular cloud Sgr B2 (Revnivtsev et al. 2004b). 


\subsection{Transients}

A significant fraction of Galactic LMXBs are transients. During outbursts their luminosities can change by more than 6-7 orders of magnitude (e.g. Tanaka \& Shibazaki 1996). The "on" state of bright X-ray transients typically lasts several weeks, but due to their extreme brightness at that time, they may still be detected on a sky map averaged over several years even if they were undetectable most of the time.

It is usually considered that the observed X-ray brightness variations in LMXB transients are caused by a developed mass transfer instability in the accretion disk (e.g. Meyer \& Meyer-Hofmeister 1984; Lasota 2001). In this case, the X-ray luminosity observed from such a source at any given time does not provide us with information about the global mass transfer rate in the binary system, which is of interest to us. The global mass transfer rate could be determined by averaging the X-ray luminosity of a transient source over a period that is much longer than the accretion disk mass accumulation time; however, this time scale might be too long (e.g. Chen et al. 1997) to be testable by available X-ray observations. The inclusion of transient sources with their fluxes averaged over some arbitrary period (not related to any physically motivated time scale) might distort the true distribution of the mass transfer rates in which we are interested.

Therefore, in our analysis we treated the transient sources in two ways: 1) we included all sources detected at the 5-year averaged INTEGRAL/IBIS map of the Galactic center; and 2) we filtered out all transient sources from our sample. In the latter case, we adopted the following selection criterium for separating transient sources from persistent ones: a transient should have the ratio of maximal 3-day averaged flux to the all-time mean flux of more than 5.0. We note that transient nature is difficult to ascertain for weaker sources because they only become detectable after accumulating a large part of the total exposure time. Therefore, our resulting sample of transient sources is likely to be significantly incomplete at low fluxes.

The final list of accepted sources is presented in Table 1. The map of the Galactic bulge region with the sources from Table 1 is presented in Fig. 1.

\subsection{Distances to the sources}

The distances to the majority of our sources are unknown. However, assuming that LMXBs are distributed in the Galactic bulge similarly to ordinary stars, we can calculate the density distribution of LMXBs in the bulge and predict the spread of values of the flux-to-luminosity conversion coefficient for our sources. This determines the typical systematic error we are making in estimating source luminosities by assuming that all sources are at the same, $8 \mathrm{kpc}$, distance from the Sun.

To this end, we adopted a mass model of the Galaxy consisting of the Galactic bulge, Galactic disk, and nuclear stellar disk (e.g. Launhardt et al. 2002). We did not consider the nuclear stellar cluster, which is important in the innermost $\sim 10^{\prime}$ of the Galaxy, because its size is smaller than the angular resolution of INTEGRAL/IBIS.

The stellar density in the bulge was assumed to follow model G3 from Dwek et al. (1995). We adopted the mass of the bulge to be $1.3 \times 10^{10} M_{\odot}$ (Dwek et al. 1995). The stellar density in the Galactic disk is

$\rho_{\text {disk }}(r, z)=\rho_{0, \text { disk }} \exp \left[-\left(\frac{r_{\mathrm{m}}}{r}\right)^{3}-\left(\frac{r}{r_{\text {disk }}}\right)-\left(\frac{|z|}{z_{\text {disk }}}\right)\right]$,
Table 1. List of known or likely LMXB sources (persistent and transients) in the Galactic bulge region detected by INTEGRAL on the time-average map.

\begin{tabular}{|c|c|c|c|}
\hline \# & Name & $\begin{array}{c}\text { Flux, mCrab } \\
17-60 \mathrm{keV}\end{array}$ & $\begin{array}{c}\text { Flux }^{b}, \mathrm{mCrab} \\
2-10 \mathrm{keV}\end{array}$ \\
\hline 1 & GRS 1758-258 & $59.07 \pm 0.07$ & 25 \\
\hline 2 & GX 1+4 & $54.14 \pm 0.08$ & 11 \\
\hline 3 & GX 5-1 & $48.14 \pm 0.07$ & 972 \\
\hline 4 & GX 354-0 & $37.57 \pm 0.08$ & 99 \\
\hline 5 & 1E $1740.7-2942$ & $31.79 \pm 0.07$ & 10 \\
\hline 6 & 4U 1724-30 & $17.14 \pm 0.07$ & 25 \\
\hline 7 & A1742-294 & $13.71 \pm 0.07$ & $18[1]$ \\
\hline 8 & GX9+1 & $16.50 \pm 0.09$ & 532 \\
\hline 9 & SLX 1735-269 & $11.21 \pm 0.07$ & 14 \\
\hline 10 & GX3+1 & $9.93 \pm 0.07$ & 300 \\
\hline 11 & SLX1744-299 & $5.47 \pm 0.07$ & $9.0[1]$ \\
\hline 12 & 1E1742.8-2853 & $6.29 \pm 0.07$ & \\
\hline 13 & 1E1743.1-2843 & $5.20 \pm 0.07$ & $8.1[2]$ \\
\hline 14 & SLX 1737-282 & $3.94 \pm 0.07$ & $5.4[3]$ \\
\hline 15 & SLX1744-300 & $2.73 \pm 0.07$ & $5.1[1]$ \\
\hline 16 & IGR J17254-3257 & $1.72 \pm 0.08$ & 3.5 \\
\hline 17 & IGR J17475-2253 & $1.09 \pm 0.08$ & \\
\hline 18 & IGR J17353-3539 & $0.88 \pm 0.08$ & \\
\hline 19 & IGR J17505-2644 & $0.81 \pm 0.07$ & \\
\hline 20 & IGR J17585-3057 & $0.81 \pm 0.07$ & \\
\hline 21 & IGR J17586-2129 & $0.73 \pm 0.08$ & \\
\hline 22 & IGR J17448-3231 & $0.70 \pm 0.07$ & \\
\hline \multicolumn{4}{|c|}{ Transients detected on the averaged map } \\
\hline 1 & GRS 1741.9-2853 & $3.17 \pm 0.07$ & \\
\hline 2 & SAX J1747.0-2853 & $3.30 \pm 0.07$ & \\
\hline 3 & KS 1741-293 & $4.95 \pm 0.07$ & \\
\hline 4 & XTE J1739-285 & $2.21 \pm 0.07$ & \\
\hline 5 & GRS 1747-313 & $1.53 \pm 0.07$ & \\
\hline 6 & IGR J17464-3213 & $22.07 \pm 0.07$ & \\
\hline 7 & SLX 1746-331 & $0.69 \pm 0.07$ & \\
\hline 8 & IGR J17353-3257 & $1.12 \pm 0.07$ & \\
\hline 9 & MXB 1730-33 & $4.20 \pm 0.08$ & \\
\hline 10 & IGR J17331-2406 & $1.01 \pm 0.08$ & \\
\hline 11 & XTE J1720-318 & $1.73 \pm 0.08$ & \\
\hline 12 & IGR J17597-2201 & $4.62 \pm 0.08$ & \\
\hline 13 & XTE J1817-330 & $7.01 \pm 0.09$ & \\
\hline 14 & 4U 1746-37 & $2.77 \pm 0.09$ & \\
\hline 15 & 4U 1705-32 & $2.30 \pm 0.09$ & \\
\hline 16 & IGR J17091-3624 & $4.75 \pm 0.10$ & \\
\hline
\end{tabular}

${ }^{a}$ The $1 \mathrm{mCrab}$ flux in the 17-60 keV energy band for a Crab-like spectrum corresponds to an energy flux of $\sim 1.4 \times 10^{-11} \mathrm{erg} / \mathrm{s} \mathrm{cm}^{-2}$ and to a luminosity $1.1 \times 10^{35} \mathrm{erg} / \mathrm{s}$ for a distance of $8 \mathrm{kpc}$.

${ }^{b}$ Fluxes in the $2-10 \mathrm{keV}$ energy band reported for some sources in the last column were obtained from RXTE/ASM measurements or adopted from the literature if a reference is given. The hard X-ray fluxes of the sources SLX 1744-299 and SLX 1744-300, which are not resolvable with INTEGRAL/IBIS, were calculated from their measured summed flux by assuming their flux ratio to be 2:1 (Sidoli et al. 1999).

(1) Sidoli et al. (1999); (2) Porquet et al. (2003); (3) in't Zand et al. (2002).

where $r_{\text {disk }}=2.2 \mathrm{kpc}, r_{\mathrm{m}}=2.5 \mathrm{kpc}, z_{\text {disk }}=130 \mathrm{pc}$ (Revnivtsev $\&$ Sazonov 2007). The total mass of the Galactic stellar disk was taken to be $2.5 \times 10^{10} M_{\odot}$.

The nuclear stellar disk (NSD) was assumed to have the density distribution ( $r$ and $z$ are measured in parsecs)

$\rho_{\mathrm{NSD}}=\rho_{\mathrm{d}} r^{-\alpha} \mathrm{e}^{-|z| / z_{\mathrm{d}}}$

where $z_{\mathrm{d}}=45 \mathrm{pc}$. At $r<120 \mathrm{pc}$ the slope $\alpha=0.1$ and the normalization constant $\rho_{\mathrm{d}}=300 M_{\odot} / \mathrm{pc}^{3}$. At $120 \mathrm{pc}<r<220 \mathrm{pc}$, $\alpha=3.5$, and at $r>220 \mathrm{pc}, \alpha=10$ (Launhardt et al. 2002), and 


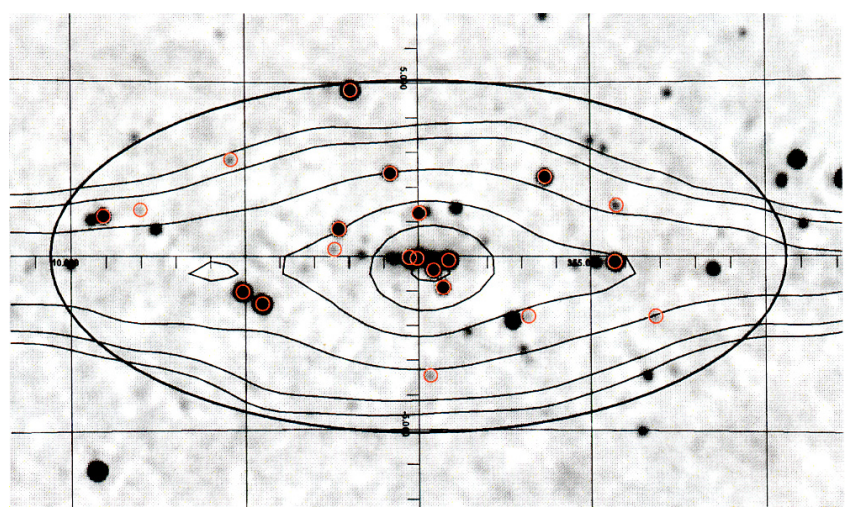

Fig. 1. Map of the Galactic center region in the 17-60 keV energy band obtained by INTEGRAL/IBIS. The ellipse encloses the region of our study. Sources used in the analysis of the LMXB LF are marked by circles. Contours are isophotes of the $4.9 \mu \mathrm{m}$ surface brightness of the Galaxy (COBE/DIRBE) demonstrating the bulge/disk structure of the inner Galaxy.

we adjust the constant $\rho_{\mathrm{d}}$ so that the density distribution is continuous. The total adopted mass of the NSD is $1.4 \times 10^{9} M_{\odot}$. In reality this quantity is uncertain by some $50 \%$ (Launhardt et al. 2002).

Based on this model, we determined the distribution of stars over the distance from the Sun in the region of our study (ellipse $\left.|l|<10.7^{\circ},|b|<5.1^{\circ}\right)$. The ratio $(D / 8 \mathrm{kpc})^{2}$ defines the luminosity correction factor $C F$. The probability density of this correction factor can be calculated from the probability density $P(D)$ of the source distance, which can be found by integrating the distribution of stellar mass $\rho_{*}(l, b, D)$ in a given direction $(l, b)$ over the solid angle $\Omega$ of our study:

$P(D)=\frac{\int_{\Omega} \rho_{*}(l, b, D) D^{2} \mathrm{~d} \Omega}{\int_{\Omega} \int_{D=0}^{D=\infty} \rho_{*}(l, b, D) D^{2} \mathrm{~d} \Omega \mathrm{d} D}$.

The probability density of $C F$ is presented in Fig. 2. It turns out that $\log _{10} C F<0.3$ for $80 \%$ of sources. Therefore, assuming that all bulge sources are located at the same distance $(8 \mathrm{kpc})$ from the Sun, we can predict the luminosities of most sources to within a factor 2 , this uncertainty being comparable to expected variations in source fluxes.

\subsection{Spectral corrections}

Previously, for constructing the luminosity function of LMXBs in galaxies one usually used instruments operating in the standard X-ray energy band, 2-10 keV, or similar bands (Grimm et al. 2002; Gilfanov 2004). The INTEGRAL/IBIS sky survey is done in the hard X-ray band, $17-60 \mathrm{keV}$. Therefore, to compare the LMXB LF derived here with those reported before, we should take the spectral shapes of LMXBs into account in the broad energy range $1-100 \mathrm{keV}$.

It is well-known that the brightest LMXBs emit most of their luminosity in the standard X-ray band $(\sim 2-10 \mathrm{keV})$, while less luminous objects emit similar amounts of energy in the standard and hard X-ray $(\sim 10-100 \mathrm{keV})$ bands. Bright LMXBs usually have spectra with an exponential cutoff at energies of 6-10 keV (e.g. Rappaport et al. 1969; Toor et al. 1970; Mitsuda et al. 1984; Pavlinsky et al. 1994) and essentially do not continue into the hard X-ray band (e.g. Gilfanov et al. 1993). In contrast, dim LMXBs are characterized by a powerlaw spectral shape between 2 and $60 \mathrm{keV}$ (Mitsuda et al. 1989;

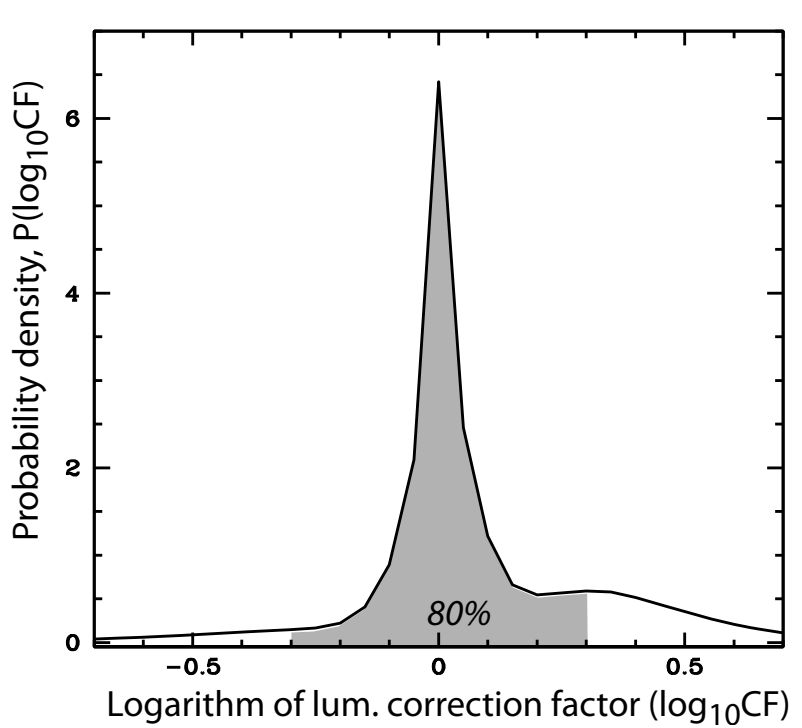

Fig. 2. Probability density of the luminosity correction factor. The probability density is normalized so that $\int P\left(\log _{10} C F\right) \mathrm{d}\left(\log _{10} C F\right)=1$. Eighty per cent of sources in the studied region of the Galaxy are expected to fall into the interval shown in gray.

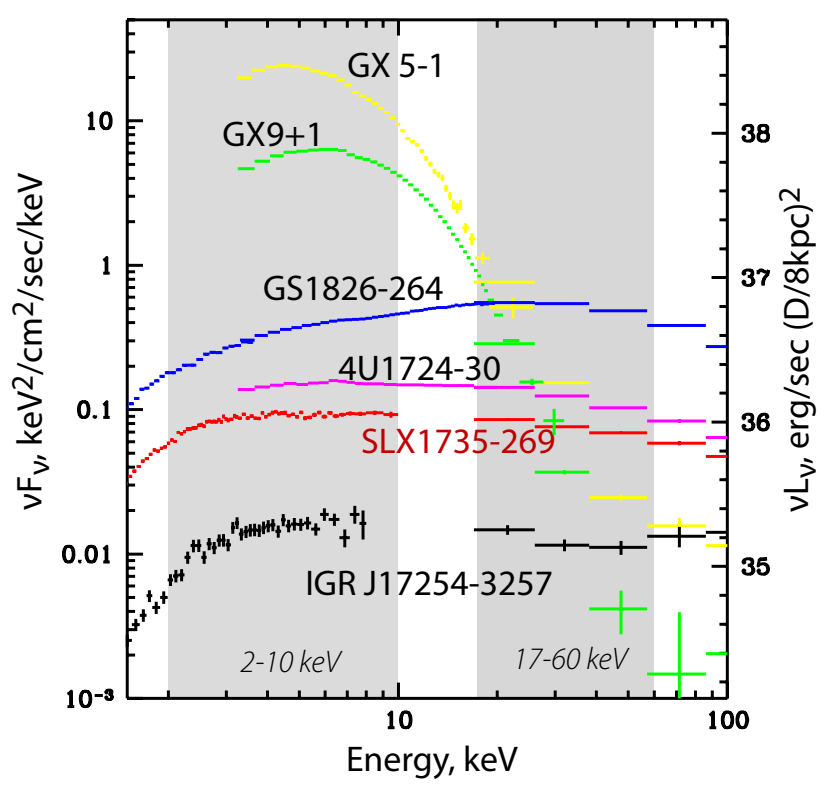

Fig. 3. Typical broadband spectra of LMXBs. The right axis shows the logarithm of the luminosity, assuming a distance of $8 \mathrm{kpc}$ to all sources. The gray stripes denote the energy bands under discussion: $17-60 \mathrm{keV}$ (the INTEGRAL/IBIS energy band) and 2-10 keV (the standard X-ray band).

Grebenev et al. 1991; Barret et al. 1991; Zhang et al. 1996; Barret et al. 2000). We can therefore expect that the LMXB LF constructed in the 17-60 keV energy band should strongly differ from that derived in the standard X-ray band.

To demonstrate this, we constructed the broadband spectra of Galactic bulge sources with different luminosities using all available instruments - INTEGRAL/IBIS, RXTE/PCA, Swift/XRT, ASCA/GIS. This allowed us to cover the wide energy range 1-100 keV (see Fig. 3). The LMXB GS 1826-24 formally lies outside the elliptical region of our study, but since its distance from the Sun is similar to those of bulge sources (e.g. in 't Zand et al. 1999). Its broadband spectrum has very high statistics, and this spectrum is also shown in the figure. 
One can see dramatic changes in the broadband behavior of source spectra at a threshold luminosity of $\sim$ a few $\times 10^{37} \mathrm{erg} / \mathrm{s}$. At high luminosities, most of the energy is emitted below $17 \mathrm{keV}$. At low luminosities, the spectral shape in the $2-60 \mathrm{keV}$ band is close to a power law with photon index $\Gamma \sim 1.9-2$, and similar fluxes are emitted at $2-10 \mathrm{keV}$ and $17-60 \mathrm{keV}$.

The time-averaged fluxes of most of the brightest sources in the $2-10 \mathrm{keV}$ band are directly measured by the All-Sky Monitor aboard RXTE, and we present these measurements in Table 1. Based on these data and Fig. 3, we adopted the following spectral correction factors for the subsequent analysis:

$\frac{L_{2-10 \mathrm{keV}}}{L_{17-60 \mathrm{keV}}}=\left\{\begin{array}{c}40 \text { if } L_{2-10 \mathrm{keV}}>2 \times 10^{37} \mathrm{erg} / \mathrm{s} \\ 1.25 \text { if } L_{2-10 \mathrm{keV}}<2 \times 10^{37} \mathrm{erg} / \mathrm{s} .\end{array}\right.$

In comparing below the LMXB LFs in the $2-10 \mathrm{keV}$ and 17-60 keV energy bands, we mostly used direct RXTE/ASM measurements to determine the $2-10 \mathrm{keV}$ luminosities for sources with $L_{2-10 \mathrm{keV}} \gtrsim 10^{36} \mathrm{erg} / \mathrm{s}$, and assumed that those sources with luminosities below this RXTE/ASM completeness limit are in the hard spectral state.

It is important to note that the spectral correction factors introduced above may not be applicable to rare, extremely luminous LMXBs such as the Galactic source GRS 1915+105, which is more powerful that any source in our Galactic bulge sample and is apparently in a highly unusual spectral state.

\section{Results}

\subsection{Luminosity function of faint $L M X B S$}

The luminosity functions of all and only persistent LMXBs detected by INTEGRAL/IBIS in the Galactic bulge region are shown in Fig. 4. These LFs are defined so as to give the number of sources within the studied region containing $1.65 \times 10^{10} M_{\odot}$ of stars.

It can be seen that the LF of weak LMXBs $\left(L_{17-60 \mathrm{keV}}<\right.$ $10^{36.5} \mathrm{erg} / \mathrm{s}$ ) is relatively flat. The LF of all LMXBs can be approximated by the function $L_{35} \mathrm{~d} N / \mathrm{d} L_{35}=(12 \pm 4) L_{35}^{-(0.13 \pm 0.13)}$ in the range $10^{34.7} \mathrm{erg} / \mathrm{s}<L_{17-60 \mathrm{keV}}<10^{36.5} \mathrm{erg} / \mathrm{s}$, and the LF of peristent sources can be approximated by the function $L_{35} \mathrm{~d} N / \mathrm{d} L_{35}=(4.7 \pm 2.2) L_{35}^{(0.04 \pm 0.2)}$. Here, $L_{35}-$ is the hard X-ray (17-60 keV) luminosity in units of $10^{35} \mathrm{erg} / \mathrm{s}$.

There are no objects with $L_{17-60 \mathrm{keV}}>10^{37} \mathrm{erg} \mathrm{s}^{-1}$ in our sample. Consequently, there is an abrupt drop in the number density of very bright sources, as can be seen in Fig. 4. In fact, there is only one non-transient (but strongly variable) source in the whole Milky Way that has a higher luminosity: GRS 1915+105 with $L_{17-60 \mathrm{keV}} \sim 5 \times 10^{37} \mathrm{erg} / \mathrm{s}$. This value was calculated from the time-averaged flux of the source of $261 \mathrm{mCrab}$ in the energy band 17-60 keV from Krivonos et al. (2007b), assuming an $11 \mathrm{kpc}$ source distance, Harlaftis \& Greiner (2004). Since such ultraluminous sources can be easily detected with INTEGRAL throughout the Galaxy, we can estimate their expected number in the Galactic bulge region studied here by considering that this region contains $16 \%$ of the total stellar mass of the Galaxy.

As the ratio of the luminosities of faint $\left(L_{2-10 \mathrm{keV}} \lesssim 2 \times\right.$ $\left.10^{37} \mathrm{erg} / \mathrm{s}\right)$ sources in the hard $(17-60 \mathrm{keV})$ and standard $(2-10 \mathrm{keV}) \mathrm{X}$-ray bands does not depend on luminosity and is about unity (namely, we assume 1.25), our measurements sug-

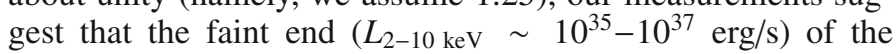
LMXB LF should be similalry flat in the $2-10 \mathrm{keV}$ energy band: $\mathrm{d} \log N / \mathrm{d} \log L \sim-1$.

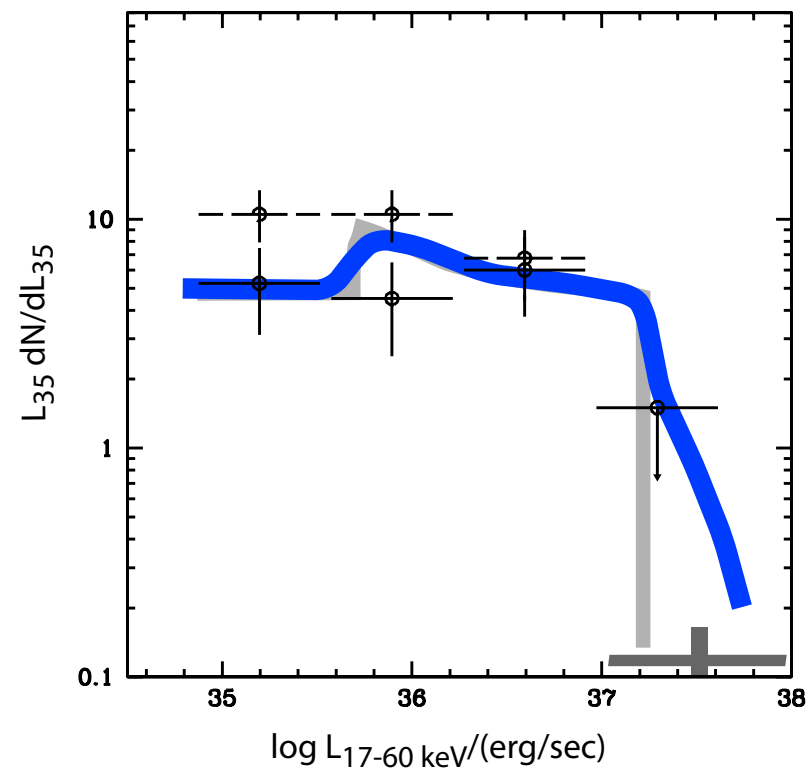

Fig. 4. Luminosity function of LMXBs detected by INTEGRAL/IBIS in the Galactic bulge region. The LF of persistent sources is shown by solid crosses, and that of all sources (including transients) by dashed crosses. The thick gray cross represents an estimate of the number of LMXBs with extremely high luminosities based on the single such source in the Galaxy, GRS $1915+105\left(L_{17-60 \mathrm{keV}} \sim 5 \times 10^{37} \mathrm{erg} / \mathrm{s}\right)$. This estimate takes into account that the bulge region covered by the present study contains only $16 \%$ of the total stellar mass of the Galaxy. The thick gray line is the analytic model of the LMXB LF from Gilfanov (2004) normalized to $1.65 \times 10^{10} M_{\odot}-$ the stellar mass within the considered region, recalculated into the $17-60 \mathrm{keV}$ energy band using the introduced spectral correction factors and multiplied by an additional factor of 0.4 . The thick blue line is the same LF model convolved with the distribution of the luminosity correction factor, which is important for sources in our sample due to unknown values of exact distances (see Fig. 2).

To compare the LMXB LF derived here in the $17-60 \mathrm{keV}$ energy band with the analytic approximation of the $2-10 \mathrm{keV}$ LF presented by Gilfanov (2004), (which has a slope $\mathrm{d} \log N / \mathrm{d} \log L \sim-1$ at its faint end, i.e. at $L_{2-10 \mathrm{keV}} \lesssim 2 \times$ $10^{37} \mathrm{erg} / \mathrm{s}$ ), we recalculated the latter into the $17-60 \mathrm{keV}$ energy band using the spectral correction factors described above. This predicted LF, rescaled to the stellar mass contained in the considered region $\left(1.65 \times 10^{10} M_{\odot}\right)$, is shown in Fig. 4 by the thick gray line. The thick blue line is the same LF model convolved with the distribution of the luminosity correction factor, which is important for sources in our sample due to unknown values of exact distances. These curves were additionally multiplied by a factor of 0.4 in order to match the normalization of the LF measured with INTEGRAL in the Galactic bulge. We note that a similar indication of the underabundance of LMXBs in the Milky Way as compared to other galaxies was previousely reported by Gilfanov (2004). It should be mentioned that this difference might be partly caused by the absence of transients in our sample, while Gilfanov (2004) considered all sources detected in galaxies.

The analytical model of Gilfanov (2004) is in good agreement with the hard X-ray LF measured in the present study (see Fig. 4). The sharp drop of the hard X-ray LF at luminosities $L_{17-60 \mathrm{keV}} \gtrsim 10^{37} \mathrm{erg} / \mathrm{s}$ can be explained naturally by the sudden spectral change, as sources with $L_{2-10 \mathrm{keV}} \lesssim 2 \times 10^{37} \mathrm{erg} / \mathrm{s}$ ) have very soft spectra and therefore very low luminosities in hard X-rays). At lower luminosities, the shape of the hard X-ray 
LF closely corresponds to that of the LMXB LF in the 2-10 keV energy band.

As is apparent from Fig. 4, transient sources provide a significant contribution to the LMXB LF. If we describe the LF by the function $L_{35} \mathrm{~d} N / \mathrm{d} L_{35}=$ const. in the luminosity range $10^{34.7}-10^{36.5} \mathrm{erg} / \mathrm{s}$, the normalization increases by a factor of $\sim 1.7$ after adding the transient sources.

We should note that, despite the low star-formation rate in the Galactic bulge, some bulge HMXBs may be present in our sample (see e.g. estimates of the star formation rate in the Galactic center region in Mezger \& Pauls 1979; Figer et al. 2004). This can be especially important at the lowest luminosities because the LF of HMXBs is much steeper than for LMXBs below $\sim 2 \times 10^{37} \mathrm{erg} / \mathrm{s}$ (see e.g., Grimm et al. 2002; Gilfanov 2004), and HMXBs can appear as a numerous population of X-ray sources at low luminosities (see e.g. Pfahl et al. 2002). For example, even for a star formation rate as low as $\dot{M}_{*}=0.03 M_{\odot} /$ year, the number of HMXBs with luminosities $10^{35}-10^{36} \mathrm{erg} / \mathrm{s}$ will be comparable to that of all similarly faint sources in the region of our study. Therefore, the true number of faint LMXBs will be even lower than suggested by the derived luminosity function.

\subsection{Possible interpretations of the break in the $L F$}

Our findings indicate, that at relatively low hard X-ray luminosities $\left(L_{17-60 \mathrm{keV}} \lesssim 2 \times 10^{37} \mathrm{erg} / \mathrm{s}\right)$, the luminosity function of LMXBs has a flat slope $\mathrm{d} \log N / \mathrm{d} \log L \sim-1$. At higher luminosities, there is a very pronounced drop in the LF. This can be explained by the high luminosity sources typically having very soft spectra and thus emitting a very small part of their energy in hard X-rays.

On the other hand, due to the almost one-to-one correspondence between the luminosities of faint LMXBs $\left(10^{35}-10^{37} \mathrm{erg} / \mathrm{s}\right)$ in the hard $(17-60 \mathrm{keV})$ and standard $(2-10 \mathrm{keV})$ energy bands, the flat slope of the hard X-ray LF simply translates into the flat slope of the $2-10 \mathrm{keV} \mathrm{LF}$.

We can thus conclude that after taking the spectral corrections into account, the shape of the hard X-ray LF of Galactic bulge LMXBs measured in this work is broadly compatible with the 2-10 keV LMXB LF of Gilfanov (2004), which is flat at luminosities $L_{2-10 \mathrm{keV}}<2 \times 10^{37} \mathrm{erg} / \mathrm{s}$. Our results are inconsistent with the assumption that the LF of LMXBs in the standard X-ray band at luminosities $L_{2-10 \mathrm{keV}}=10^{35}-10^{37} \mathrm{erg} / \mathrm{s}$ has the same slope $\mathrm{d} \log N / \mathrm{d} \log L \sim-1.8$ as in the luminosity range $L_{2-10 \mathrm{keV}}=10^{37.3}-10^{38} \mathrm{erg} / \mathrm{s}$.

The flattening of the LMXB LF at luminosities $L_{2-10 \mathrm{keV}} \lesssim$ $2 \times 10^{37} \mathrm{erg} / \mathrm{s}$ (Gilfanov 2004; Voss \& Gilfanov 2006) with respect to the steep slope at higher luminosities could be due to various reasons: i) a break in the distribution over mass transfer rates from the companion star; ii) a change in the correspondence between the mass transfer rate in the binary system and the mass accretion rate onto the compact object, possibly associated with the spectral state transition. We now briefly comment on each of these possibilities.

\subsubsection{State transition}

It is interesting that the break in the LMXB LF (in standard $\mathrm{X}$-ray energy band) occurs almost exactly at the luminosity at which the sources experience a spectral state transition. At high luminosities, practically all sources are in the so-called soft/high spectral state and emit most of their luminosity in the optically thick regime. At low luminosities, the sources are in the socalled low/hard spectral state, emit in the optically thin regime, and have hard spectra.

It is usually assumed that, in the soft/high spectral state, the $\mathrm{X}$-ray luminosity is directly proportional to the mass accretion rate in the binary system (e.g. Shakura \& Syunyaev 1973). The dependence of X-ray luminosity on mass accretion rate in the low/hard state is not clear. In the framework of the radiatively inefficient accretion flow models (Ichimaru 1977; Torbett 1984; Narayan \& Yi 1994; Blandford \& Begelman 1999), the accretion flow X-ray luminosity in the hard state may be a nonlinear function of the mass accretion rate at the outer edge of the accretion flow: $L_{\mathrm{x}} \propto \dot{M}^{\beta}$, where $\beta>1$. Some observational data favor such a dependence. See Gallo et al. (2006) where it is suggested that at lower mass accretion rates a progressively greater fraction of the accretion power can be carried by a jet, rather then converted into radiation.

In this case, even if the distribution of mass transfer rates in binary systems has no peculiarities at the rates corresponding to X-ray luminosities $\sim$ a few $\times 10^{37} \mathrm{erg} / \mathrm{s}$, one would still observe a break in the $\mathrm{X}$-ray luminosity function due to the change in the $L_{\mathrm{x}}-\dot{M}$ relation. If the underlying distribution of mass transfer rates has the slope $\mathrm{d} \log N / \mathrm{d} \log \dot{M}=-\alpha$ and $L_{\mathrm{x}} \propto \dot{M}^{\beta}$, then the resulting slope of the LF will be $\gamma=(\alpha+\beta-1) / \beta$. Assuming that the true mass transfer rate distribution has the slope $\alpha=1.8-2.0$, as seen at $\operatorname{luminosities} \log L_{\mathrm{x}} \sim 37.5-38.5$ (Gilfanov 2004), and that $\beta=3.3$ in the case of advection dominated accretion flow (Beckert \& Duschl 2002), the slope of the LF will be $\gamma \sim 1.2-1.3$. Such a flattening of the LF is sufficient to at least partly explain the observed behavior of the LF in the 2-10 keV energy band.

The majority of the considered X-ray sources are probably accreting neutron stars. They have solid surfaces, which can radiate the energy advected to it by the accretion flow, and the efficiency of conversion of mass transfer rate to X-ray luminosity is difficult to predict in this case. However, some mechanisms for reducing the X-ray emissivity of accreting neutron star systems might be considered (see e.g., Torbett 1984; Blandford \& Begelman 1999).

\subsubsection{Change of the binary braking mechanism}

Following Postnov \& Kuranov (2005), the break in the LMXB LF can be interpreted as the luminosity at which the mass transfer rate in the binary systems starts to be driven by the gravitational braking mechanism rather than by magnetic stellar wind braking.

If this is indeed the case, we can anticipate that the majority of such faint LMXBs should have very low masses for optical companions $\left(M_{\mathrm{opt}} \lesssim 0.2-0.4 M_{\odot}\right.$, see Postnov \& Kuranov 2005) and short orbital periods $(<2-3 \mathrm{~h})$, and be very faint in the optical and near-infrared spectral bands: the observable brightness of sources could be as low as $m_{\mathrm{R}}>20$ even without any interstellar extinction (see estimates of the optical brightness of compact binary systems in van Paradijs \& McClintock 1994). These predictions can be directly checked when accurate localizations of such X-ray sources are achieved with the help of X-ray telescopes. Positional accuracy of the order of few arcseconds or less is necessary in this case, because the spatial density of such faint optical objects $\left(m_{\mathrm{R}}>20-22\right)$ is very high in the direction of the Galactic bulge. 


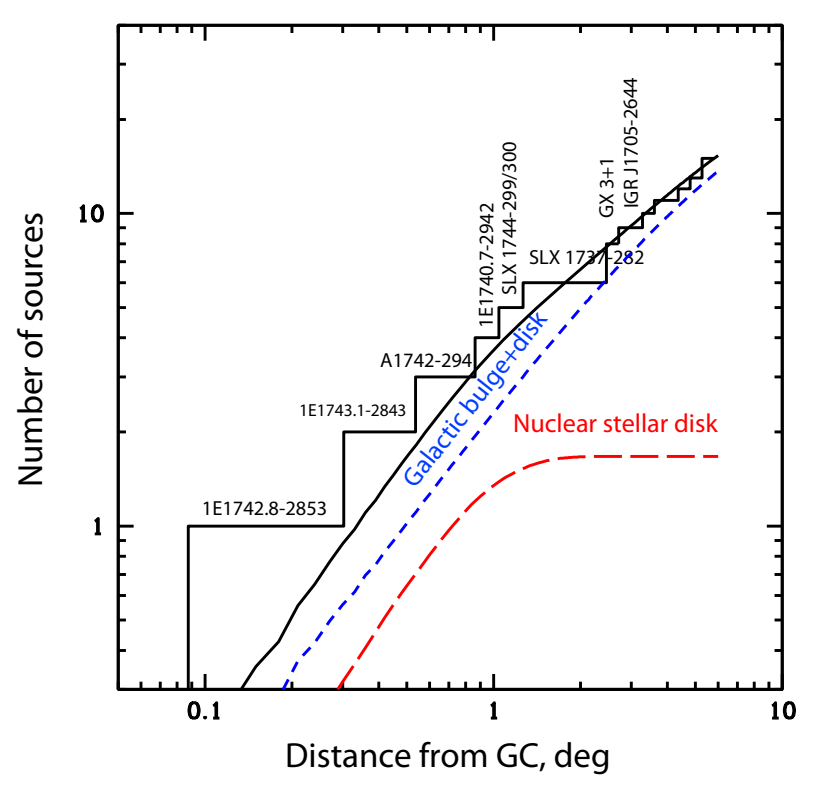

Fig. 5. Cumulative number of persistent LMXBs in the Milky Way bulge seen by INTEGRAL/IBIS within the circular region of a given projected radii. The solid curve shows the number of sources predicted by the Galaxy mass model described above and the LMXB LF of Gilfanov (2004) (multiplied by 0.4). The contributions of the Galactic bulge and nuclear stellar disk components are indicated by the shortand long-dashed lines, respectively. For the model curve we assumed the luminosity limit of $10^{35} \mathrm{erg} / \mathrm{s}$. Positions of sources in the central region of the bulge are labeled.

\subsection{Distribution of $L M X B$ s in the Galactic center region}

\subsubsection{Persistent sources}

An extensive study of LMXB distributions in the Milky Way and in other galaxies has shown that their number density closely traces the stellar mass distribution (e.g. Gursky \& Schreier 1975; Skinner 1993; Grebenev et al. 1996; Grimm et al. 2002; Gilfanov 2004). A rough comparison of the surface density of LMXBs in the Galactic bulge region with that of stars was previously done by Skinner (1993) based on archival observations with different instruments and by Grebenev et al. (1996) based on a smaller sample of sources detected with the ART-P telescope aboard the GRANAT observatory. Now, after using the sample of sources detected by INTEGRAL over five years of its operation, we can make a somewhat better comparison.

The cumulative angular distribution of the number of persistent LMXBs detected by INTEGRAL in the Galactic bulge region as a function of projected angular distance from the Galactic center is shown in Fig. 5 by the histogram. To match the number of observed sources at distances $<5.1^{\circ}$ from the Galactic center with the galactic mass model described above (the total stellar mass contained in this region is $\approx 1.2 \times 10^{10} M_{\odot}$ ) and the average LMXB LF of Gilfanov (2004), the latter was multiplied by a factor of 0.4 .

Interestingly, the radial distribution of sources indicates an excess in the number of LMXBs inside the innermost $\sim 2^{\circ}$ with respect to what is expected from the distribution of stars in the Galactic bulge. Note that a similar effect was previously observed by Skinner 1993. The excess itself has low statistical significance; instead of the predicted $\sim 1$ source within the innermost $0.6^{\circ}$ around Sgr $\mathrm{A}^{*}$, we see three sources, but it is consistent with the presence of LMXBs from the so-called nuclear

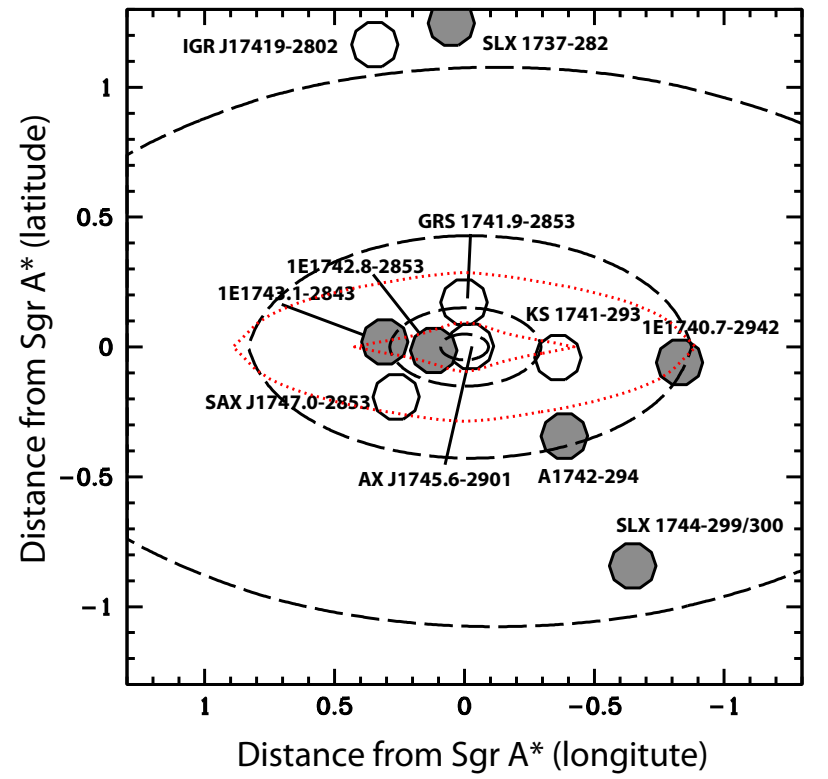

Fig. 6. Positions of all sources detected by IBIS/ISGRI within the inner $1.3^{\circ}$ around the center of the Galaxy $\left(\mathrm{Sgr} \mathrm{A}^{*}\right)$. Dashed (black) curves are contours of the $i$ so surface density of the stellar mass in the model of the Galaxy, which includes the bulge, the nuclear stellar disk and nuclear stellar cluster (which is important only within $\sim 10^{\prime}$ around Sgr A*). Dotted curves are iso surface density contours of the nuclear stellar disk component only. Filled (gray) circles show positions of persistent sources, open circles the positions of transient ones.

stellar disk (e.g. Launhardt et al. 2002). This allows us to tentatively suggest that at least the innermost sources, namely 1E1742.9-2853 and 1E1743-2843, reside in the nuclear stellar disk.

To visualize the distribution of sources in the innermost region of the Galaxy (see Fig. 6), we present the positions of detected LMXBs within $1.3^{\circ}$ around Sgr A* with overlayed contours of $i$ so surface density of stars in the region for the different components of the Galactic stellar population.

It is necessary to note that the best-fit LF normalization for the Galactic bulge population of LMXBs is $\approx 0.4$ of the average over a number of nearby galaxies (Gilfanov 2004). Nevertheless, the Galactic bulge normalization is within the spread of best-fit values obtained for different galaxies (Gilfanov 2004).

\subsubsection{Transients}

Using the long time span of the INTEGRAL observations, we have the ability to effectively determine the "transientness" of sources and study these transient sources separately. In particular, it is interesting to analyze their spatial distribution in view of the predictions of some theories that the densest central regions of galactic bulges might contain increased numbers of transients due to their enhanced dynamical formation (e.g. Voss \& Gilfanov 2007).

The list of transient sources detected by INTEGRAL within the region of our study is presented in Table 2 along with their distances from the Galactic center. The cumulative number of these sources as a function of their distance from the Galactic center is shown in Fig. 7, with the cumulative mass of stars within the considered region, normalized to the number of transients at $<5.1^{\circ}$. One can note that there is a slight indication that transient sources are more concentrated towards the Galactic 
Table 2. List of transients seen by INTEGRAL/IBIS over the period 2003-2007.

\begin{tabular}{rcc}
\hline \hline$\#$ & Dist from Sgr A*, deg & Name \\
\hline 1 & 0.02 & AX J1745.6-2901 \\
2 & 0.17 & GRS 1741.9-2853 \\
3 & 0.33 & SAX J1747.0-2853 \\
4 & 0.36 & KS1 741-293 \\
5 & 1.27 & IGR J17419-2802 \\
6 & 1.36 & XTE J1739-285 \\
7 & 1.51 & GRS 1736-297 \\
8 & 2.02 & XTE J1751-305 \\
9 & 2.54 & GRS 1747-313 \\
10 & 3.22 & IGR J17464-3213 \\
11 & 4.29 & SLX 1746-331 \\
12 & 4.48 & XTE J1807-294 $17353-3257$ \\
13 & 4.67 & MXB 1730-33 \\
14 & 5.11 & IGR J17331-2406 \\
15 & 5.64 & XTE J1720-318 \\
16 & 6.18 & A 1744-361 \\
17 & 7.14 & IGR J17597-2201 \\
18 & 7.68 & XTE J1817-330 \\
19 & 7.95 & 4U 1746-37 \\
20 & 8.10 & XTE J1818-245 \\
21 & 8.56 & 4U 1705-32 1745-203 \\
22 & 8.57 & IGR J17091-3624 \\
23 & 8.68 & \\
24 & 10.60 & \\
25 & 10.66 &
\end{tabular}

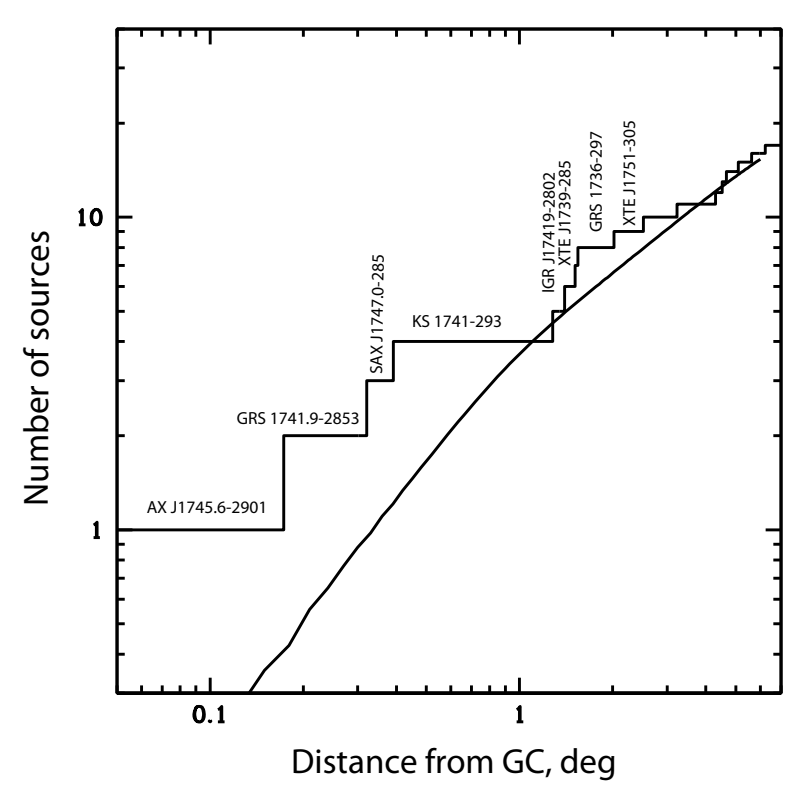

Fig. 7. Cumulative number of transient LMXB sources in the Galactic center region, as seen by INTEGRAL/IBIS. The solid line represents the adopted mass model of the Galaxy normalized to the number of sources within $5.1^{\circ}$ of the Galactic center. The positions of sources in the innermost region of the bulge are labeled.

center than the persistent ones, but the statistical significance of this effect is low. We should note that a similar effect was observed by Skinner (1993), since the majority of the sources considered in that work were transients detected with different instruments over a long period. If the effect of the increased surface density of transient sources in the center of the Galaxy is real, this might be related to the mechanism of dymanical formation of LMXBs proposed by Voss \& Gilfanov (2007).

\section{Conclusions}

We analyzed the luminosity function and spatial distribution of low-mass X-ray binaries in the central part of our Galaxy (Galactic bulge) using data from the INTEGRAL observatory in the 17-60 keV energy band, which allowed us to obtain the largest sample of LMXB and LMXB candidates in the Galactic bulge region. We have found that:

1. No sources in the considered region demonstrate hard X-ray $(17-60 \mathrm{keV})$ luminosities higher than $L_{17-60 \mathrm{keV}} \sim$ $10^{37} \mathrm{erg} / \mathrm{s}$. This happens because: a) sources with high bolometric luminosities have soft spectra with only a small portion of the bolometric luminosity emitted in the hard X-ray band, b) those sources with hard spectra do not have high bolometric luminosities. In fact, in our Galaxy there is only one source with hard X-ray luminosity as high as $L_{17-60 \mathrm{keV}} \sim 5 \times 10^{37} \mathrm{erg} / \mathrm{s}-$ GRS $1915+105$, which is pecular in many respects.

2. The luminosity function of persistent LMXBs is fairly flat at the faint end $\left(L_{17-60 \mathrm{keV}} \lesssim 10^{37} \mathrm{erg} / \mathrm{s}\right)$ : the slope is $\mathrm{d} \log N / \mathrm{d} \log L=-0.96 \pm 0.20$. At these low luminosities, the spectra of LMXBs between 2 and $60 \mathrm{keV}$ are typically similar to that of the Crab, so that nearly equal amounts of energy are released in the standard $(2-10 \mathrm{keV})$ and hard $\mathrm{X}$-ray $(17-60 \mathrm{keV})$ bands. This allows us to conclude that the LMXB LF is similarly flat in the $2-10 \mathrm{keV}$ band in the range $10^{35}-10^{37} \mathrm{erg} / \mathrm{s}$; i.e. it is significantly flatter than at higher luminosities, where $\mathrm{d} \log N / \mathrm{d} \log L \sim-1.8$. The measured shape of the hard X-ray LF of the Galactic bulge LMXBs is broadly consistent with that of the LMXB LF of Gilfanov (2004), when allowance is made of the spectral corrections, and thus confirms the flattening of the LF at $L_{2-10 \mathrm{keV}} \sim 10^{37} \mathrm{erg} / \mathrm{s}$ proposed earlier by Primini et al. (1993) and Gilfanov (2004).

3. The origin of the observed flattening in the LF is not clear. One of the possibilities is that it is connected with a change of the mechanism of removal of the angular momentum of the binary system: from magnetic stellar wind braking to gravitational braking (see Postnov \& Kuranov 2005). However, a similarly strong flattening of the LMXB LF might be caused by a change in the mass accretion rate to $\mathrm{X}$-ray luminosity conversion, as expected in radiatively inefficient accretion flow models.

4. The cumulative angular distribution of the persistent LMXBs in the Galactic center region traces the cumulative number of stars in the model consisting of the Galactic bulge and nuclear stellar disk components. The data favor (though with low statistical significance) the presence of a nuclear stellar disk component in the distribution of LMXBs (specifically, 3 sources are observed within $1.5^{\circ}$ of the Galactic center, whereas the model without the nuclear disk component predicts just $\sim 1$ source).

5. The cumulative angular distribution of the transient LMXBs differs slightly from that of the persistent sources, indicating some increase in the fraction of transient sources in the innermost regions of the Galaxy. However, the statistical significance of this increase is low $(\sim 2$ more transients within $1^{\circ}$ of Galactic center are observed than expected based on the observed distribution of persistent sources).

Acknowledgements. This research made use of data obtained from the High Energy Astrophysics Science Archive Research Center Online Service, provided by the NASA/Goddard Space Flight Center. This work was supported by DFGSchwerpunktprogramme (SPP 1177), by DFG cluster of excellence "Origin and Structure of the Universe", by grants of Russian Foundation of Basic Research 
07-02-01051, 07-02-00961-a, NSh-5579.2008.2 and program of Presidium of RAS "The origin and evolution of stars and galaxies" (P04). A.A.L. thanks International Space Science Institute (ISSI) for their hospitality during his work on the paper.

\section{References}

Barret, D., Mereghetti, S., Roques, J. P., et al. 1991, ApJ, 379, L21

Barret, D., Olive, J. F., Boirin, L., et al. 2000, ApJ, 533, 329

Beckert, T., \& Duschl, W. J. 2002, A\&A, 387, 422

Bélanger, G., Goldwurm, A., Renaud, M., et al. 2006, ApJ, 636, 275

Belczynski, K., Kalogera, V., Rasio, F. A., et al. 2008, ApJS, 174, 223

Blandford, R. D., \& Begelman, M. C. 1999, MNRAS, 303, L1

Chen, W., Shrader, C. R., \& Livio, M. 1997, ApJ, 491, 312

Dehnen, W., \& Binney, J. 1998, MNRAS, 294, 429

Dwek, E., Arendt, R. G., Hauser, M. G., et al. 1995, ApJ, 445, 716

Figer, D. F., Rich, R. M., Kim, S. S., Morris, M., \& Serabyn, E. 2004, ApJ, 601, 319

Gallo, E., Fender, R. P., Miller-Jones, J. C. A., et al. 2006, MNRAS, 370, 1351

Gilfanov, M. 2004, MNRAS, 349, 146

Gilfanov, M., Churazov, E., Sunyaev, R., et al. 1993, ApJ, 418, 844

Grebenev, S. A., Syunyaev, R. A., Pavlinskii, M. N., \& Dekhanov, I. A. 1991, SvAL, 17, 413

Grebenev, S. A., Pavlinsky, M. N., \& Sunyaev, R. A. 1996, International Conference on X-ray Astronomy and Astrophysics: Röntgenstrahlung from the Universe, 141

Grimm, H.-J., Gilfanov, M., \& Sunyaev, R. 2002, A\&A, 391, 923

Gursky, H., \& Schreier, E. 1975, IAUS, 67, 413

Harlaftis, E. T., \& Greiner, J. 2004, A\&A, 414, L13

Ichimaru, S. 1977, ApJ, 214, 840

in 't Zand, J. J. M., Heise, J., Kuulkers, E., et al. 1999, A\&A, 347, 891 in't Zand, J. J. M., Verbunt, F., Kuulkers, E., et al. 2002, A\&A, 389, L43 Kim, D.-W., Fabbiano, G., Kalogera, V., et al. 2006, ApJ, 652, 1090

Kong, A. K. H., DiStefano, R., Garcia, M. R., \& Greiner, J. 2003, ApJ, 585, 298 Krivonos, R., Revnivtsev, M., Churazov, E., et al. 2007a, A\&A, 463, 957

Krivonos, R., Revnivtsev, M., Lutovinov, A., et al. 2007b, A\&A, 475, 775

Kuulkers, E., Shaw, S. E., Paizis, A., et al. 2007, A\&A, 466, 595

Lasota, J.-P. 2001, NewAR, 45, 449
Launhardt, R., Zylka, R., \& Mezger, P. G. 2002, A\&A, 384, 112

Lipunov, V. M., Postnov, K. A., Prokhorov, M. E., \& Bogomazov, A. I. 2007, [arXiv: 0704.1387]

Lutovinov, A., Revnivtsev, M., Gilfanov, M., et al. 2005, A\&A, 444, 821

Lutovinov, A., Revnivtsev, M., Gilfanov, M., \& Sunayev, R. 2007, Proc. 6th INTEGRAL Workshop (ESA Publishing), SP-662, 241

Meyer, F., \& Meyer-Hofmeister, E. 1984, A\&A, 132, 143

Mezger, P. G., \& Pauls, T. 1979, IAUS, 84, 357

Mitsuda, K., Inoue, H., Koyama, K., et al. 1984, PASJ, 36, 741

Mitsuda, K., Inoue, H., Nakamura, N., \& Tanaka, Y. 1989, PASJ, 41, 97

Narayan, R., \& Yi, I. 1994, ApJ, 428, L13

Pavlinsky, M. N., Grebenev, S. A., \& Sunyaev, R. A. 1994, ApJ, 425, 110

Pfahl, E., Rappaport, S., \& Podsiadlowski, P. 2002, ApJ, 573, 283

Porquet, D., Rodriguez, J., Corbel, S., et al. 2003, A\&A, 406, 299

Postnov, K. A., \& Kuranov, A. G. 2005, AstL, 31, 7

Primini, F. A., Forman, W., \& Jones, C. 1993, ApJ, 410, 615

Rappaport, S., Bradt, H. V., Naranan, S., \& Spada, G. 1969, Nature, 221, 428

Revnivtsev, M., \& Sazonov, S. 2007, A\&A, 471, 159

Revnivtsev, M. G., Sunyaev, R. A., Varshalovich, D. A., et al. 2004a, AstL, 30, 382

Revnivtsev, M. G., Churazov, E. M., Sazonov, S. Yu., et al. 2004b, A\&A, 425, L49

Shakura, N. I., \& Syunyaev, R. A. 1973, A\&A, 24, 337

Sidoli, L., Mereghetti, S., Israel, G. L., et al. 1999, ApJ, 525, 215

Skinner, G. K. 1993, A\&AS, 97, 149

Tanaka, Y., \& Shibazaki, N. 1996, ARA\&A, 34, 607

van den Heuvel, E. P. J. 1975, ApJ, 198, L109

van Paradijs, J., \& McClintock, J. E. 1994, A\&A, 290, 133

van Speybroeck, L., Epstein, A., Forman, W., et al. 1979, ApJ, 234, L45

Toor, A., Seward, F. D., Cathey, L. R., \& Kunkel, W. E. 1970, ApJ, 160, 209

Torbett, M. V. 1984, ApJ, 278, 318

Trinchieri, G., \& Fabbiano, G. 1991, ApJ, 382, 82

Voges, W., Aschenbach, B., Boller, Th., et al. 1999, A\&A, 349, 389

Voss, R., \& Gilfanov, M. 2006, A\&A, 447, 71

Voss, R., \& Gilfanov, M. 2007, A\&A, 468, 49

Webbink, R. F., Rappaport, S., \& Savonije, G. J. 1983, ApJ, 270, 678

Winkler, C., Courvoisier, T. J.-L., Di Cocco, G., et al. 2003, A\&A, 411, L1

Zhang, S. N., Harmon, B. A., Paciesas, W. S., et al. 1996, A\&AS, 120, 279 